\title{
Design and Performance Evaluation of a Time Domain Microwave Imaging System
}

\author{
Xuezhi Zeng, ${ }^{1}$ Andreas Fhager, ${ }^{1}$ Peter Linner, ${ }^{2}$ Mikael Persson, ${ }^{1}$ and Herbert Zirath ${ }^{2}$ \\ ${ }^{1}$ Department of Signals and Systems, Chalmers University of Technology, 412-96 Gothenburg, Sweden \\ ${ }^{2}$ Microwave Electronics Laboratory, Chalmers University of Technology, 412-96 Gothenburg, Sweden \\ Correspondence should be addressed to Xuezhi Zeng; xuezhi@chalmers.se
}

Received 13 March 2013; Revised 17 July 2013; Accepted 15 August 2013

Academic Editor: Safieddin Safavi-Naeini

Copyright (C) 2013 Xuezhi Zeng et al. This is an open access article distributed under the Creative Commons Attribution License, which permits unrestricted use, distribution, and reproduction in any medium, provided the original work is properly cited.

\begin{abstract}
We design a time domain microwave system dedicated to medical imaging. The measurement accuracy of the system, that is, signalto-noise ratio, due to voltage noise and timing noise, is evaluated. Particularly, the effect of coupling media on the measurement accuracy is investigated both numerically and experimentally. The results suggest that the use of suitable coupling media betters the measurement accuracy in the frequency range of interest. A signal-to-noise ratio higher than $30 \mathrm{~dB}$ is achievable in the range of $500 \mathrm{MHz}$ to $3 \mathrm{GHz}$ when the effective sampling rate is $50 \mathrm{Gsa} / \mathrm{s}$. It is also indicated that the effect of the timing jitter on the strongest received signal is comparable to that of the voltage noise.
\end{abstract}

\section{Introduction}

As a potential technology for object detection and identification, ultrawideband (UWB) microwave imaging for medical applications has been a subject of extensive research in the past few years [1-6].

Microwave imaging is carried out by sending microwave signals into an object under examination and receiving reflected or scattered fields. The received signals are processed to produce an image of the object. The object response under the microwave excitation is dependent on the illuminating frequency; therefore more information about the object's property can be collected with a UWB illumination in comparison with a monofrequency case. Moreover, the conflict between spatial resolution and penetration depth, which is the technical challenge for monofrequency microwave imaging, can be resolved by using UWB technology.

Most UWB microwave imaging systems are based on commercial instruments, for example, a vector network analyzer (VNA) [1-4], a sampling or a high-speed real-time oscilloscope $[5,6]$. While these instruments provide ready solutions for experimental purposes, their high cost and massive size make these solutions not applicable for practical use. Therefore, it is desirable to have a custom-designed UWB microwave imaging system with compact size, low cost, and high speed.

In comparison with a stepped-frequency technique [7], pulsed time domain measurement technology is preferable for UWB system design due to a more simple system architecture and a higher measurement speed. The generation of UWB pulses with duration less than hundred picoseconds (the power spectrum covers the band of interest for biomedical applications) has become possible due to the fast development of advanced solid-state technology.

Despite the advantages, time domain systems have worse signal-to-noise ratios (SNRs) in comparison with frequency domain systems. A time domain system is highly likely to have poor performance if not carefully designed, particularly for a complicated measurement scenario, for example, medical imaging.

In this paper, we propose a time domain system for medical imaging. The system performance, which can be obtained under different imaging conditions, are evaluated by means of theoretical analysis and simulations. The effect of coupling media on the system performance is studied and verified experimentally. 


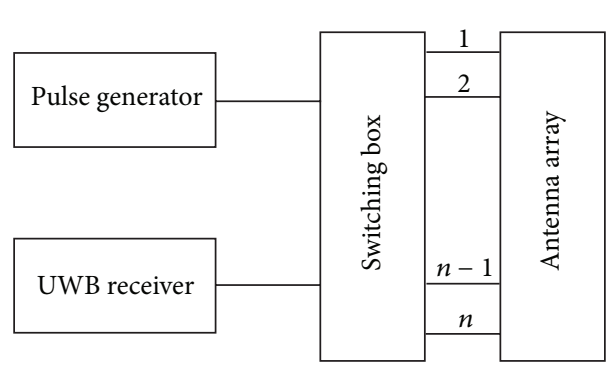

(a)

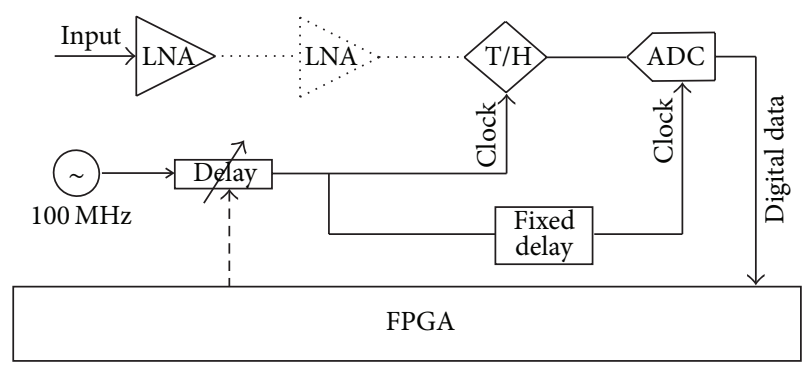

(b)

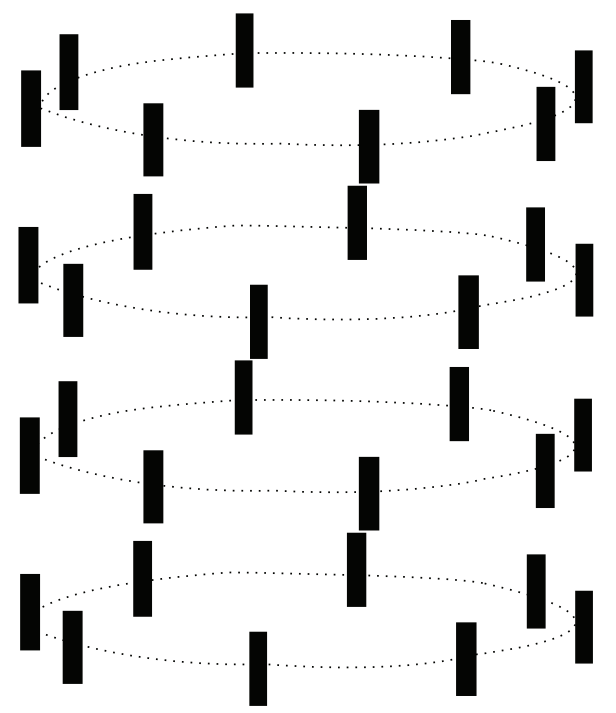

(c)

Figure 1: The proposed time domain microwave imaging system: (a) system block diagrams, (b) the hardware of the receiver, and (c) the 3D antenna array.

\section{Proposed Time Domain Microwave Medical Imaging System}

In this section, the system architecture and hardware are described in detail. Particularly, the design challenges of the receiver are discussed and a suitable solution is given.

2.1. System Block Diagram. Figure 1(a) gives the block diagram of the time domain microwave imaging system. It mainly consists of a pulse generator, a UWB receiver, an antenna array, and a switching box. The hardware of the UWB receiver is given in Figure 1(b) and the antenna array is presented in Figure 1(c).

The working principle of the system can be summarized as follows: each time one of the antennas is used to transmit a UWB pulse, which is generated by the pulse generator, into an inspected object, and scattered fields are acquired by the remaining antennas. The received signals are subsequently measured by the UWB receiver. This process is repeated until all the antennas have been used for transmitting. If there are $M$ antennas, $M \times(M-1)$ data sheets are therefore obtained. The switching box is used to select different transmitting and receiving antenna pairs.

There are mainly two different approaches for active microwave imaging: radar-based imaging and microwave tomography, which have a different frequency range of interest $[1,4]$. Our work is based on a tomographic imaging type and the frequency range of interest is from $500 \mathrm{MHz}$ to $3 \mathrm{GHz}$.

2.2. System Hardware. The system hardware is chosen considering the tradeoff between the performance, cost, and speed.

2.2.1. Pulse Generator. A commercial pulse generator from Picosecond-Pulse-Lab (PSPL) is used for pulse generation. The pulse generator has a tunable output voltage and repetition rate, therefore giving high flexibility for the system design. Figure 2 plots the output pulse of the pulse generator recorded by a sampling oscilloscope. It consists of a main pulse at about $5 \mathrm{~ns}$ and baseline perturbations appearing after $15 \mathrm{~ns}$. The full-width-half-maximum (FWHM) duration of the main pulse is about $75 \mathrm{ps}$, which gives a $3 \mathrm{~dB}$ bandwidth from $\mathrm{DC}$ to $4.2 \mathrm{GHz}$. The presence of the perturbations is due to the nonideal generation of the pulse and the perturbation level is less than $20 \%$ of that of the main pulse. In the next generation of the system, it will be replaced by an owndesigned pulse generator. 


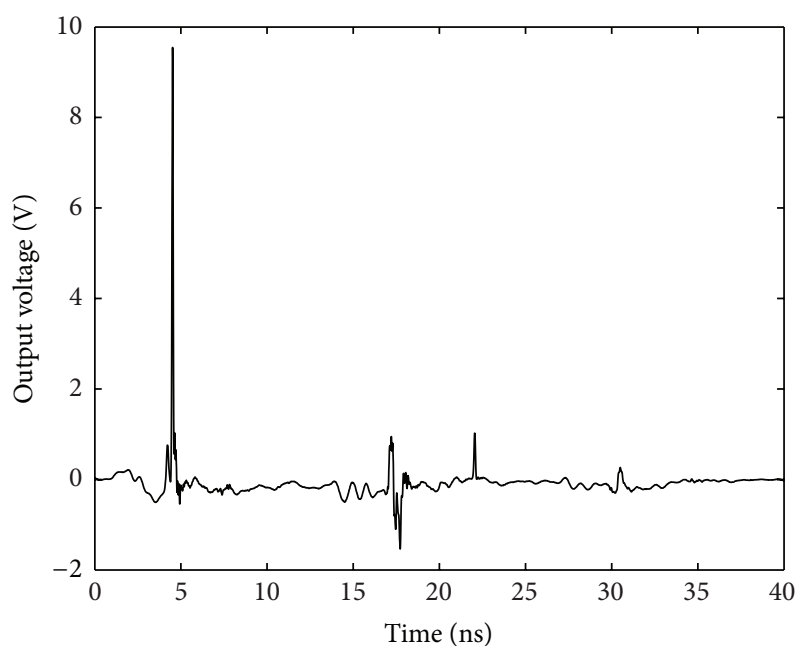

Figure 2: The maximum output voltage waveform of the impulse generator.

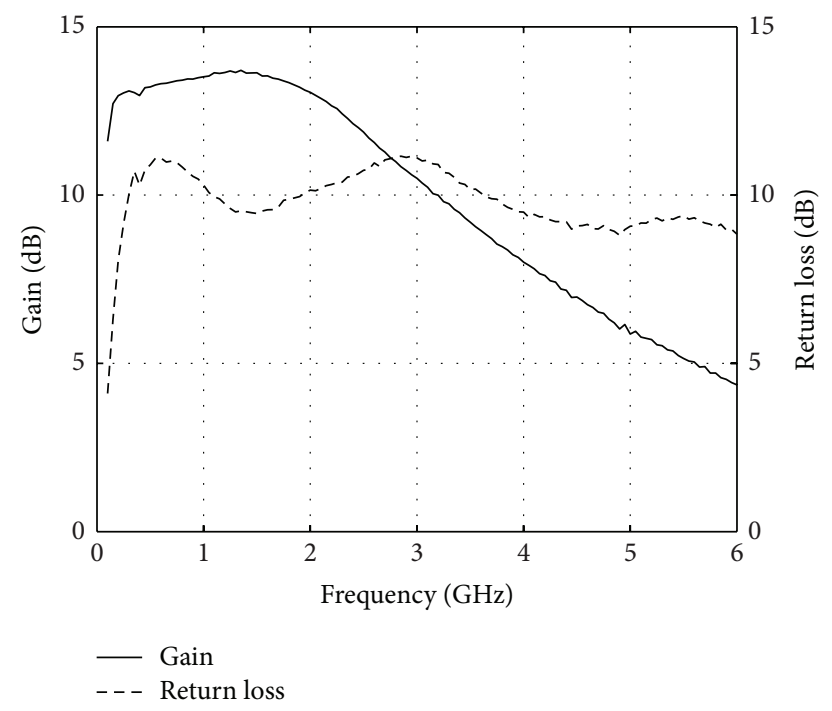

Figure 3: The return loss and gain of the LNA from $100 \mathrm{MHz}$ to $6 \mathrm{GHz}$.

2.2.2. UWB Receiver. Figure 1(b) presents the blocks of the system receiver. It is composed of a RF channel and a timing circuit module. The RF channel includes one or more low noise amplifiers (LNAs) depending on the level of the received signals, a track and hold amplifier $(\mathrm{T} / \mathrm{H})$, and an analog to digital converter (ADC). The timing circuit module is used to control the sampling and digitizing process and it consists of a highly clean external clock source (e.g., $100 \mathrm{MHz}$ ), a programmable delay chip, a fixed delay component, and a field programmable gate array (FPGA) board.

Figure 3 shows the return loss and gain of the LNA measured by using a VNA from $100 \mathrm{MHz}$ to $6 \mathrm{GHz}$. It can be seen that the LNA has a $3 \mathrm{~dB}$ bandwidth up to $3 \mathrm{GHz}$. The return loss and sampling transfer function of the $\mathrm{T} / \mathrm{H}$ are plotted in Figure 4. The return loss is measured with the VNA and the sampling transfer function is taken from the

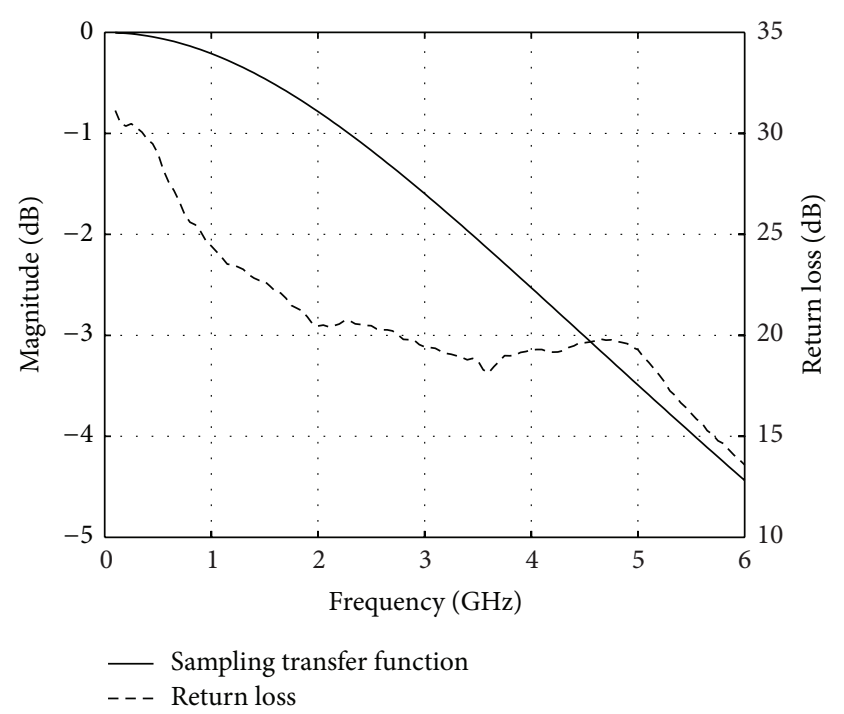

FIgURE 4: The return loss and the sampling transfer function of the $\mathrm{T} / \mathrm{H}$ from $100 \mathrm{MHz}$ to $6 \mathrm{GHz}$. The return loss is measured with the VNA and the sampling transfer function is obtained from manufacturer data.

manufacturer data. It is indicated that the $\mathrm{T} / \mathrm{H}$ is well matched to $50 \mathrm{ohm}$ in the frequency range of interest. There are many commercial ADCs which meet the system needs, and in order to facilitate the system development, an ADC from Texas Instruments is selected.

2.2.3. Antenna Array. We consider a cylindrical antenna array which consists of 32 dipoles in total, configured in four circles with eight antennas in each circle. The principal design of the antenna array under investigation can be found in Figure 1(c). The diameter of the cylindrical antenna array is $140 \mathrm{~mm}$ and the height is $120 \mathrm{~mm}$. The antenna array is either placed in the air or immersed into a coupling medium which is used to coupling more EM energy from the antenna to the human body under test. The main reason for using the dipole is its simple structure, which can be easily and accurately modeled in a computational program. In addition, dipoles can be positioned in close proximity to the imaging target, with high-element density when configured in an imaging array. Dipoles are also cheap and easy to manufacture. A switching matrix is used for changing the transmitting and receiving antennas.

2.3. Receiver Design. Analog bandwidth and sampling rate are two main designing issues for a time domain receiver. The analog bandwidth of a system is a frequency range within which the system components are well matched and the measured signal is little distorted. The sampling rate is specified by Nyquist sampling theorem. It is well known that in order to reconstruct a sampled signal, the sampling rate should be at least twice the signals bandwidth.

Ideally, the amplified UWB signal is directly fed to a wideband, high dynamic range ADC for digitization. Key limitations to this approach are that current ADCs do not 


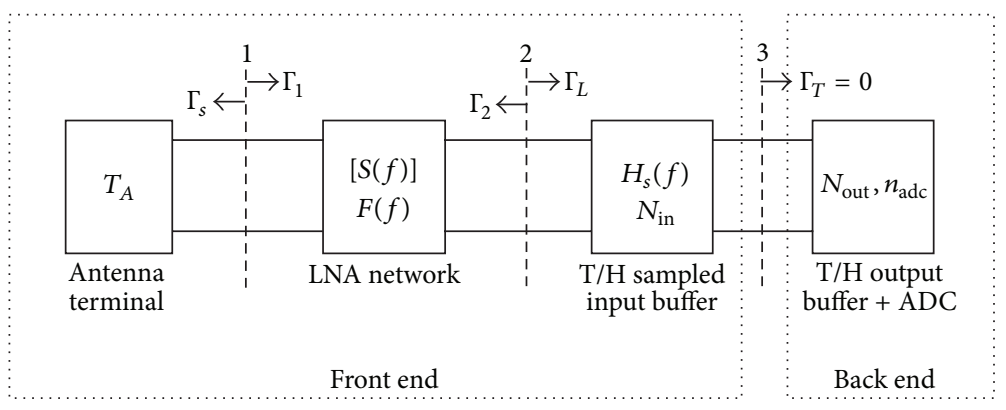

Figure 5: Block diagrams used to depict the voltage noise flow in the system.

usually have sufficient bandwidth and sampling rate for this very wideband application. High-speed ADCs commercially available generally have an analog bandwidth of a few hundred megahertz and a sampling rate of a few hundred megasamples per second, which are insufficient for the real-time acquisition of a UWB pulse with several gigahertz bandwidth. In addition, maintenance of good sampling linearity at frequencies above the UHF band is technologically challenging and most current ADCs suffer rapidly degrading linearity above gigahertz.

The limitation of the bandwidth could be overcome by using a UWB $\mathrm{T} / \mathrm{H}$ ahead of a lower speed ADC, as shown in Figure 1(b). The received signal is sampled with the $\mathrm{T} / \mathrm{H}$ and the output keeps constant for a period of time. The low bandwidth-held output waveform can then be processed by an ADC with substantially reduced bandwidth. The sampling rate problem is solved by employing an equivalent time sampling technique [8]. In each cycle of the pulse, a few samples are taken and the samples obtained from several cycles are finally assembled to reconstruct the full waveform.

In order to implement this technique, the measured signal should be repetitive. For a medical imaging application such as breast cancer detection and brain imaging, biological activities usually cause little problem for the imaging. Therefore, the object response can be assumed to be repetitive during the measurement time of interest.

The generation of an accurate sampling clock is crucial for the sampling accuracy. Recently, it has been shown that FPGA technology is capable of producing an equivalent time sampling clock [9]. However, the poor jitter performance of a FPGA generated clock would seriously degrade the measurement accuracy. Therefore, a commercial programmable delay chip is used here for producing a fine delay in each wave cycle. This delay resolution is as fine as 10 picosecond (ps) [10] which corresponds to an effective sampling rate of $100 \mathrm{GSa} / \mathrm{s}$. The propagation delay between the $\mathrm{T} / \mathrm{H}$ and the $\mathrm{ADC}$ is taken into account by using a fixed delay component. The FPGA is mainly responsible for controlling the generation of the equivalent time sampling clock and storing the digital data.

\section{Noise Analysis}

In this section, the noise sources of the proposed system are identified and their contributions to a time domain measurement are analyzed. Only random noise is considered since deterministic error can be dealt with by calibrations.

All noise present in the system can be classified as voltage noise and timing noise, which are analyzed, respectively, below. Although timing noise can be converted to voltage noise, we exclude this part of contribution from voltage noise analysis.

3.1. Voltage Noise. Figure 5 depicts the voltage noise model of the system. According to this figure, the voltage noise present in the system consists of the antenna noise, the LNA network noise, the T/H noise, and the ADC noise.

The antenna noise represents external noise intercepted by the antenna and it is characterized by the antenna noise temperature $T_{A}$, which is generally assumed to be constant in microwave frequency range.

The noise performance of the LNA is characterized by the noise figure (NF), F. For several cascaded LNAs, the effective noise figure becomes [11]

$$
F(f)=F_{1}(f)+\frac{F_{2}(f)-1}{G_{1}(f)}+\frac{F_{3}(f)-1}{G_{1}(f) \cdot G_{2}(f)}+\cdots .
$$

Figure 6 plots the LNAs NF measured by using a noise figure analyzer and the value is below $3 \mathrm{~dB}$ in the frequency range of interest. The oscillating effect at higher frequencies is the measurement uncertainty due to the decreasing gain as frequency.

The effective input noise temperature of the LNA network, $T_{g}$, can then be calculated from the NF according to the following equation [11]:

$$
T_{g}(f)=[F(f)-1] \cdot T_{0}
$$

Here $T_{0}=290 \mathrm{~K}$ is the standard room temperature.

The noise contributed by the $\mathrm{T} / \mathrm{H}$ is composed of sampled input buffer noise and output buffer amplifier noise, and their effective noise power spectral density is denoted by $N_{\text {in }}$ and $N_{\text {out }}$, respectively. The ADC's voltage noise consists of thermal noise and quantization noise, and the noise power is represented by $n_{\mathrm{adc}}$.

We classify components ahead of the sampling stage as front-end receiver and those after as back-end receiver. The front-end noise is integrated into samples during sampling process; therefore it is not affected by the presence of 


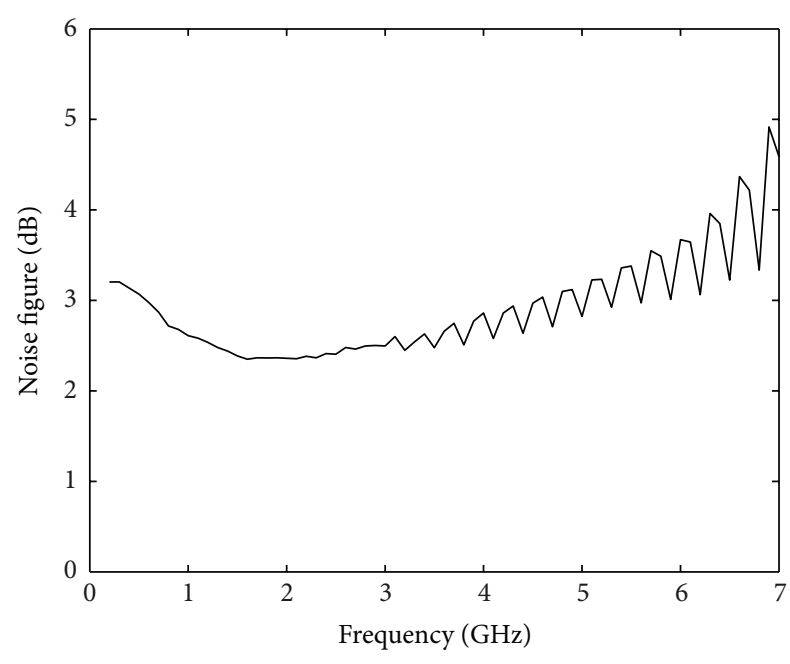

FIgURE 6: The noise figure of the LNA measured with a noise figure analyzer.

the ADC. According to the figure, the front-end noise power is the sum of three components [11]:

$$
n_{\text {front }}=n_{a}+n_{g}+n_{\mathrm{in}}
$$

where $n_{a}, n_{g}$, and $n_{\text {in }}$ represent noise contributions from the antenna, the LNA, and the sampled input buffer of the $\mathrm{T} / \mathrm{H}$, respectively.

Taking the mismatch effects into account, the noise integrated into the samples is calculated according to the following equations $[12,13]$ :

$$
\begin{gathered}
n_{a}=Z_{0} \int_{f_{1}}^{f_{2}} K \cdot T_{A} \cdot \alpha(f) \cdot \eta(f) d f, \\
n_{g}=Z_{0} \int_{f_{1}}^{f_{2}} K \cdot T_{g}(f) \cdot \alpha(f) \cdot \eta(f) d f, \\
n_{\text {in }}=\int_{f_{1}}^{f_{2}} N_{\text {in }} \cdot \eta(f) d f .
\end{gathered}
$$

Here $Z_{0}=50 \Omega$ is the load impedance, $K=1.38066 \times$ $10^{-23} \mathrm{~J} / \mathrm{K}$ is the Boltzmann constant, $f_{1}$ and $f_{2}$ define the frequency range for the noise power calculation. $\alpha$ is the ratio of the available noise power at reference plane 2 to the available power at reference plane 1 , and $\eta$ represents the ratio of the noise power delivered to the back-end receiver to that available at reference plane 2. These two ratios can be expressed in terms of the network parameters according to [14]:

$$
\begin{gathered}
\alpha(f)=\frac{\left(1-\left|\Gamma_{s}(f)\right|^{2}\right)\left|s_{21}(f)\right|^{2}}{\left|1-s_{11}(f) \Gamma_{s}(f)\right|^{2}\left(1-\left|\Gamma_{2}(f)\right|^{2}\right)}, \\
\eta(f)=\frac{\left(1-\left|\Gamma_{2}(f)\right|^{2}\right)\left|H_{s}(f)\right|^{2}}{\left|1-\Gamma_{L}(f) \Gamma_{2}(f)\right|^{2}} .
\end{gathered}
$$

Here, $\Gamma_{s}, \Gamma_{2}$, and $\Gamma_{L}$ are reflection coefficients, $s_{11}$ and $s_{21}$ are the scattering parameters of the LNA network. $H_{s}$ is

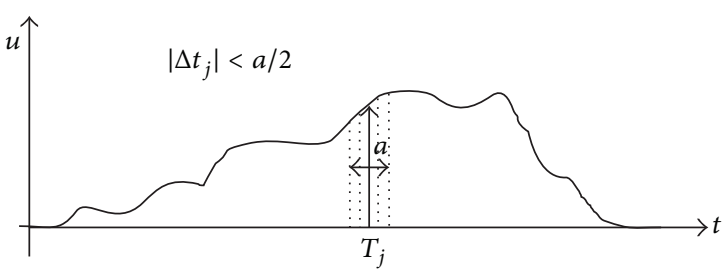

FIGURE 7: The illustration of the timing jitter effect.

the sampling transfer function, which has a single-pole low pass characteristic (Figure 4) and can be expressed in terms of its sampling bandwidth $B_{\mathrm{TH}}$ :

$$
H_{s}(f)=\frac{1}{1+j\left(f / B_{\mathrm{TH}}\right)} .
$$

In addition to the front-end noise $n_{\text {front }}$, the total system noise, $n_{\text {sys }}$, should also take into account the noise contribution from the back-end receiver, which consists of the output buffer amplifier noise, $n_{\text {out }}$, and the ADC noise, $n_{\text {adc }}[11]$ :

$$
n_{\text {sys }}=n_{\text {front }}+n_{\text {out }}+n_{\text {adc }} \text {, }
$$

where

$$
\begin{gathered}
n_{\text {out }}=N_{\text {out }} \times B_{\mathrm{adc}} \times \frac{\pi}{2}, \\
n_{\mathrm{adc}}=\frac{0.5 \times\left(0.5 \times V_{p p}^{\mathrm{adc}}\right)^{2}}{\mathrm{SNR}_{\mathrm{adc}}} .
\end{gathered}
$$

Here $B_{\text {adc }}$ is the bandwidth of the ADC, $V_{p p}^{\text {adc }}$ is the full scale input of the ADC, and $\mathrm{SNR}_{\mathrm{adc}}$ is the full scale SNR of the ADC. The $\pi / 2$ comes about because the effective noise bandwidth of the ADC is the product of its $3 \mathrm{~dB}$ bandwidth and $\pi / 2$.

3.2. Timing Jitter. Noise present at time axis is known as timing jitter. It is the undesired deviation from expected sampling instants. Jitter usually causes problems in the measurement of a time domain signal. Depending on the signals' bandwidth, slight variations in the timing may seriously degrade the SNR.

If denoting a signal under sampling by $u(t)$, then due to the presence of timing jitter, samples are taken as follows:

$$
u_{j}=u\left(T_{j}+\Delta t_{j}\right)
$$

where $u_{j}$ is the $j$ th sample, $T_{j}$ is the expected sampling instant of the $j$ th sample, and $\Delta t_{j}$ is the random deviation from the expected sampling instant, which is known as timing jitter. Figure 7 illustrates an example of the timing jitter effect.

In the proposed system, there are several contributions of the timing jitter: the jitter of the pulse generator, the jitter of the external clock source, the aperture jitter of the T/H, and the jitter of the programmable delay chip. The total timing jitter present during the sampling process can be obtained from the following equation:

$$
\delta_{j}=\sqrt{\delta_{p}^{2}+\delta_{s}^{2}+\delta_{d}^{2}+\delta_{\mathrm{TH}}^{2}},
$$


where $\delta_{p} \approx 1.5 \mathrm{ps}, \delta_{s} \approx 90 \mathrm{fs}, \delta_{d} \approx 1.2 \mathrm{ps}$, and $\delta_{\mathrm{TH}} \approx 84 \mathrm{fs}$ are the rms jitter of the pulse generator, the clock source, the programmable delay chip, and the $\mathrm{T} / \mathrm{H}$, respectively. Taking all the values into the above equation, we obtain the total timing jitter of around $2 \mathrm{ps}$ rms.

\section{Noise Performance}

SNR is used to characterize the noise performance of the time domain system. Poor SNRs may result in misdetection of objects or produce distortion to image reconstructions [15]. In this section, the systems measurement SNRs are evaluated for different conditions.

4.1. SNR Definition. The SNR of a continuous wave system is defined as the ratio of signal power-to-noise power. This definition, however, is not applicable to our time domain system since a pulse is not a power signal but an energy signal. One common SNR definition for a time domain system is the ratio of signal energy to noise power, which is usually used in a pulsed radar system. For a near field imaging system, SNR defined in frequency domain is more appropriate.

The standard deviation of a time domain signals amplitude spectrum can be calculated from several repetitive sampled noisy signals [16]:

$$
\delta(f)=\sqrt{\frac{1}{N-1} \sum_{i=1}^{N}\left(Y_{i}(f)-\overline{Y(f)}\right)^{2}} .
$$

Here $N$ is the number of repeated measurements and $Y_{i}(f)$ is the amplitude spectrum of an acquired time domain waveform of index $i . Y(f)$ is the average of the amplitude spectrums of all acquired signals. by

The SNR for each frequency component is then calculated

$$
\operatorname{SNR}(f)=\frac{\overline{Y(f)}}{\delta(f)}
$$

4.2. SNR due to Voltage Noise. As mentioned in Section 3, the measurement is affected by both the voltage noise and timing jitter. For voltage noise-resulted SNR, (12) becomes the following when $N$ goes to infinity due to the zero-mean property of the random noise:

$$
\operatorname{SNR}(f)=\frac{\left|S(f) \cdot H_{\mathrm{rcr}}(f)\right|}{\sqrt{N_{\text {sys }}(f)}},
$$

where $S$ is the frequency spectrum of the signal acquired by the antenna, $H_{\text {rcr }}$ is the receivers transfer function, and $N_{\text {sys }}$ is the power spectral density of the systems voltage noise in the unit of $\mathrm{V}^{2} / \mathrm{Hz}$.

The voltage noise does not retain its original bandwidth after being sampled, instead it is heterodyned into one Nyquist interval (e.g., $50 \mathrm{MHz}$ for a $100 \mathrm{MHz}$ sampling clock) by the sampling process. When splicing all the samples obtained from several repetitive measurements together,
TABLE 1: System components' specifications.

\begin{tabular}{lcccccc}
\hline$B_{\text {th }}$ & $\begin{array}{c}V_{p p}^{\text {th }} \\
\mathrm{GHz}\end{array}$ & $\begin{array}{c}N_{\text {in }}{ }^{1} \\
\mathrm{~V}^{2} / \mathrm{Hz}\end{array}$ & $\begin{array}{c}N_{\text {out }}{ }^{2} \\
\mathrm{~V}^{2} / \mathrm{Hz}\end{array}$ & $\begin{array}{c}B_{\mathrm{adc}} \\
\mathrm{GHz}\end{array}$ & $\begin{array}{c}V_{p p}^{\mathrm{adc}} \\
\mathrm{V}\end{array}$ & $\begin{array}{c}\mathrm{SNR}_{\mathrm{adc}}{ }^{3} \\
\mathrm{~dB}\end{array}$ \\
\hline 4.5 & 1.0 & $7.2 \times 10^{-17}$ & $4.1 \times 10^{-17}$ & 0.8 & 1.5 & 68.4 \\
\hline \\
${ }^{1}$ Given by the manufacturer. \\
${ }^{2}$ Given by the manufacturer. \\
${ }^{3}$ Specified at $20 \mathrm{MHz}$.
\end{tabular}

TABLE 2: Noise contributions from different components.

\begin{tabular}{lcccccc}
\hline RMS noise, $\mathrm{mV}$ & $n_{a}$ & $n_{g}$ & $n_{\text {in }}$ & $n_{\text {out }}$ & $n_{\text {adc }}$ & $n_{\text {sys }}$ \\
\hline Air & 0.04 & 0.04 & 0.60 & 0.23 & 0.20 & 0.68 \\
Coupling media & 0.46 & 0.44 & 0.62 & 0.23 & 0.20 & 0.94 \\
\hline
\end{tabular}

the noise is additionally processed and the time domain noise in the samples will be essentially distributed over the increased Nyquist bandwidth (half of the effective sampling rate). Therefore, the power spectral density of the voltage noise becomes

$$
N_{\text {sys }}(f)=\frac{n_{\text {sys }}}{f_{s} / 2},
$$

where $f_{s}$ is the effective sampling rate.

Based on the above analysis, the SNR of the proposed system due to voltage noise can now be evaluated. The antenna array was modeled by using a finite difference time domain (FDTD) method. The UWB pulse shown in Figure 2 was used as the transmitting signal and signals acquired by antennas were obtained by means of FDTD simulation. The air and different coupling media were considered in the evaluation.

4.2.1. Air. According to Table 1, the full scale input of the $\mathrm{T} / \mathrm{H}$ is $V_{p p}^{\text {th }}=1$ volt peak to peak. The amplified signal should be limited to this level in order to retain the good linearity of the $\mathrm{T} / \mathrm{H}$. Through calculations, we found that one LNA is enough to bring the maximum received signal to the input limit of the $\mathrm{T} / \mathrm{H}$ when the antenna array was placed in the air.

Figure 8(a) shows the strongest received signal in a $40 \mathrm{~ns}$ time window and this plot has a different starting reference time from that in Figure 2. Due to the narrow band characteristic of the dipole, the signal resembles a modulated sinusoidal. By comparing this picture with Figure 2, we could easily identify the responses corresponding to the main pulse and perturbations.

Noise contributed by different components were calculated according to (3)-(7) and the values were given in Table 2 as rms voltage. The antenna noise temperature was set to be the standard room temperature. The noise power was calculated from $f_{\min }=50 \mathrm{MHz}$ to $f_{\max }=20 \mathrm{GHz}$ and the error due to the frequency truncation is very small due to the rapidly decreasing gain of the system. In the calculation of $n_{g}$, we make the NF of the LNA; $F=1 / \alpha[12]$ when its gain is less than unity. It is seen that the system noise is dominated by the sampled input buffer noise of the $\mathrm{T} / \mathrm{H}$. The antenna noise is about the same level as the contribution from the LNA. 


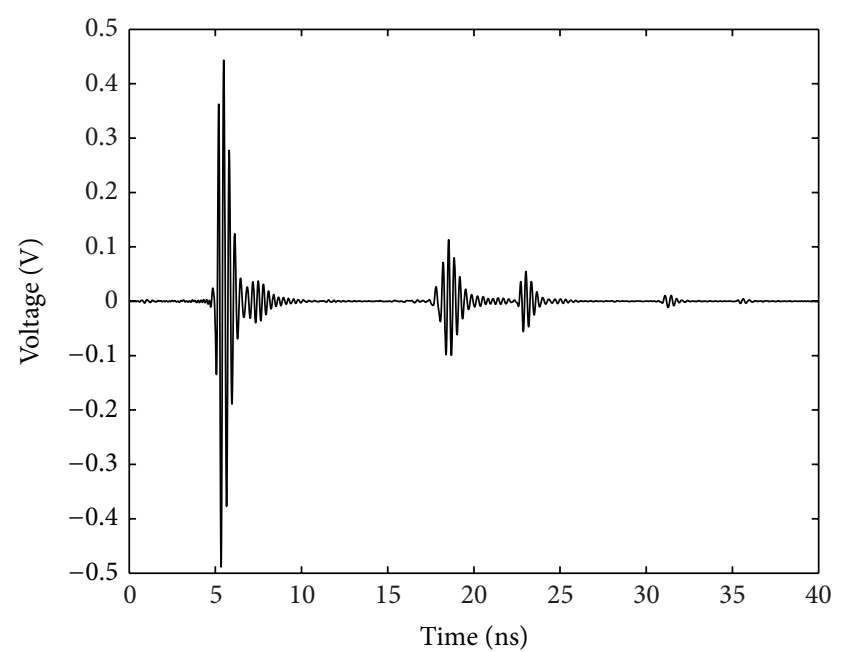

(a)

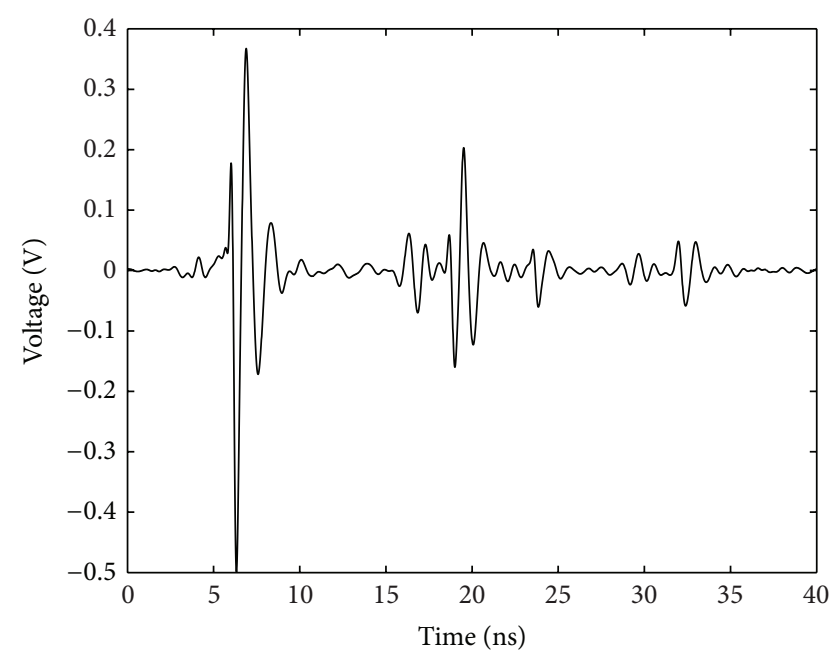

(b)

FIGURE 8: The strongest measured signals when the antenna array is (a) placed in the air and (b) immersed in coupling medium 1.

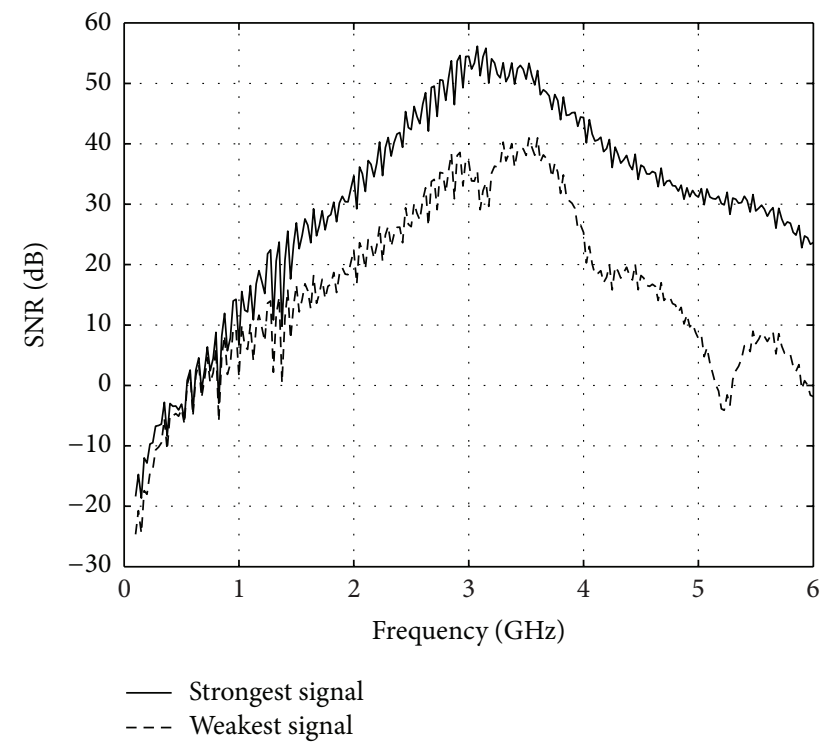

FIGURE 9: The SNR of the strongest and weakest signals when the antenna array is put in air.

The SNRs of the strongest and weakest measured signals were then calculated from (13) and shown in Figure 9. Here we assume an effective sampling rate of $50 \mathrm{Gsa} / \mathrm{s}$.

The results suggest that the optimal $3 \mathrm{GHz}$ working band of system is about from $2 \mathrm{GHz}$ to $5 \mathrm{GHz}$ when the antenna array is placed in the air. The maximum SNR difference between the strongest and the weakest signals is about $20 \mathrm{~dB}$ in this frequency range.

4.2.2. Coupling Media. In a microwave medical imaging system, a coupling medium is usually used in order to couple the EM energy from the antenna to the human body more effectively. In the FDTD program, a one-pole Debye model is

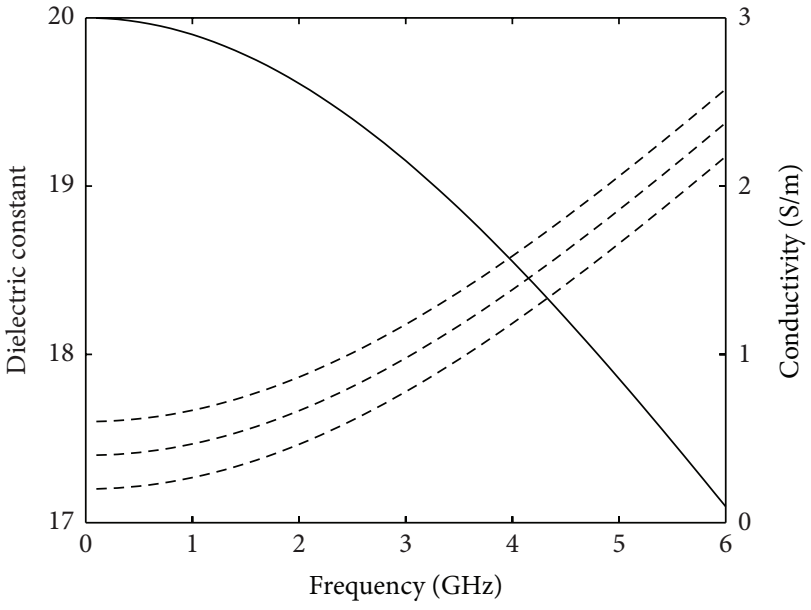

FIGURE 10: The dielectric properties of the coupling media used in the FDTD simulation. The solid line is the permittivity of the three coupling media and the dashed lines represent their conductivity values.

used to model the frequency-dependent dielectric properties of the coupling medium [17]:

$$
\epsilon(\omega)=\epsilon_{\infty}+\frac{\epsilon_{s}-\epsilon_{\infty}}{1+j \omega \tau}+\frac{\sigma_{s}}{j \omega \epsilon_{0}} .
$$

Here $\epsilon$ is the complex permittivity, $\omega$ is the angular frequency, $\epsilon_{s}, \epsilon_{\infty}, \tau$, and $\sigma_{s}$ are fitting parameters.

We consider three different coupling media which have the same permittivity but different conductivity values. Figure 10 plots the permittivity and conductivity of the coupling media. We use $\epsilon_{s}=20, \epsilon_{\infty}=5, \sigma_{s}=0.2,0.4$, and 0.6 for matching liquid 1 , coupling medium 2 , and coupling medium 3, respectively. These parameters are chosen in consideration of the dielectric properties of human breast tissue [18]. The time constant $\tau$ is determined by fitting the 


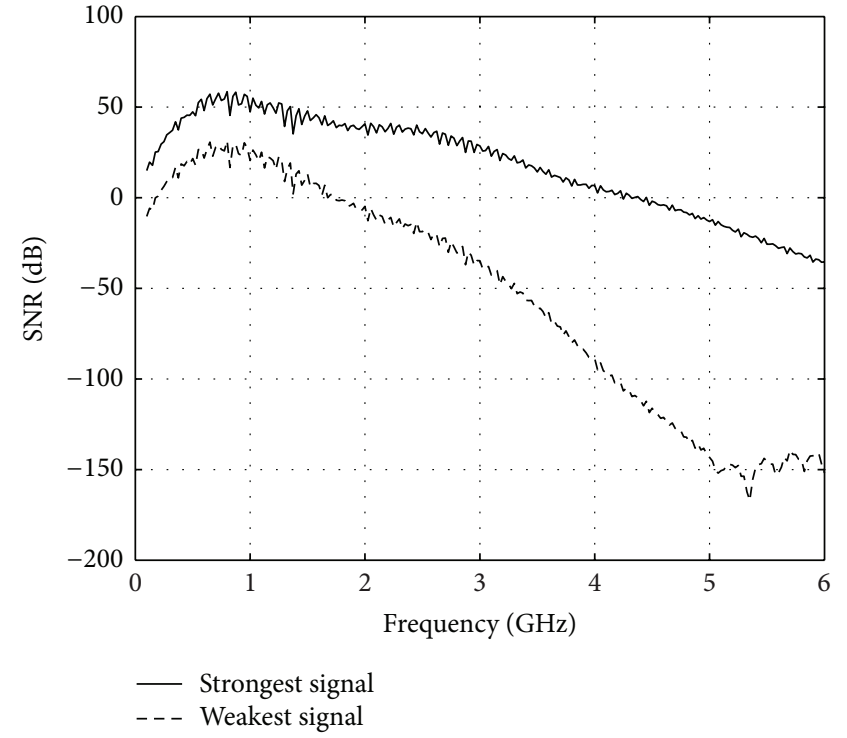

(a)

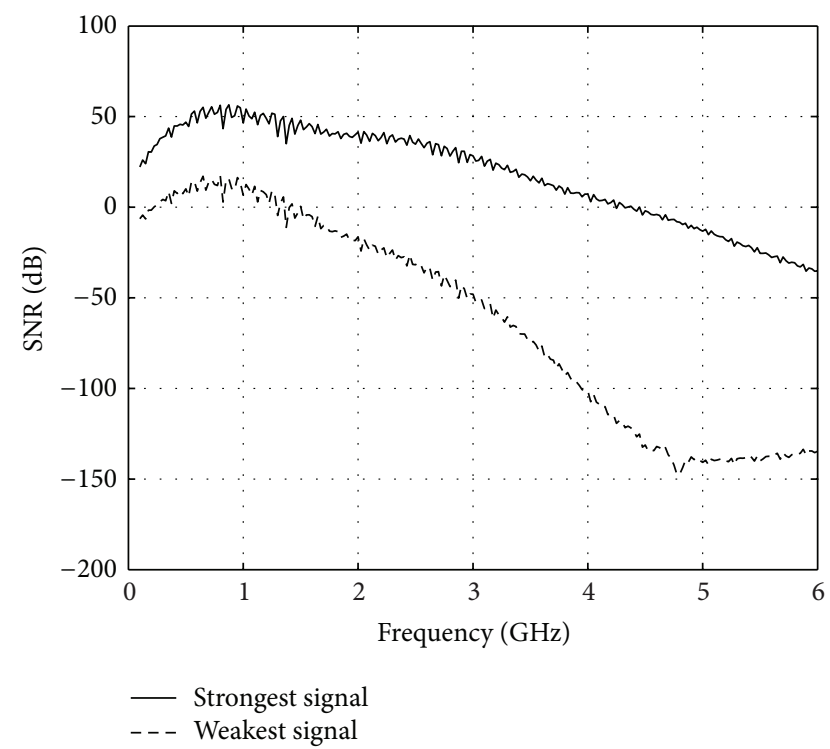

(b)

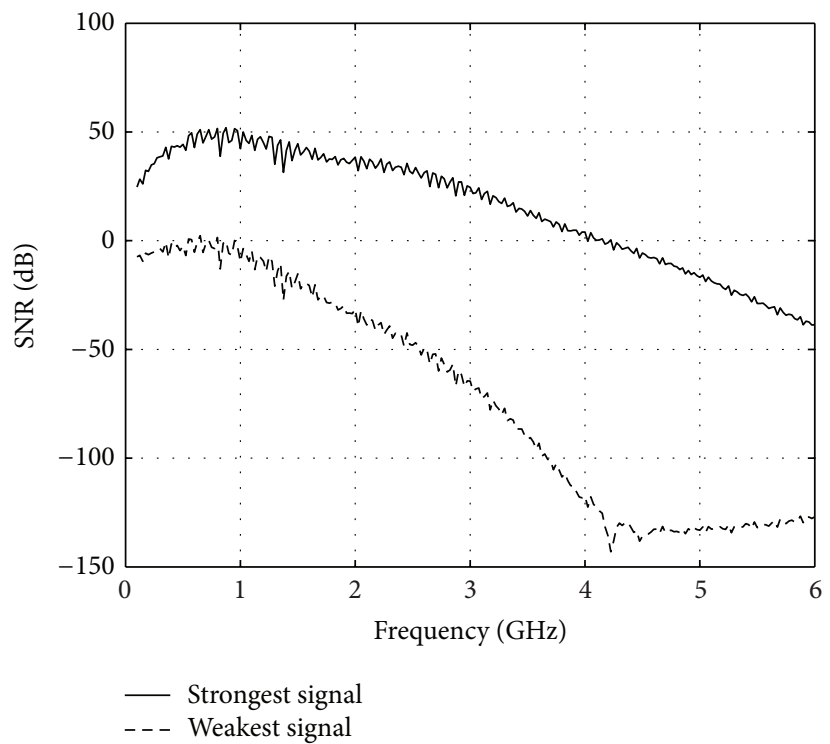

(c)

FIGURE 11: The SNRs of the strongest and weakest signals when (a) coupling medium 1, (b) coupling medium 2, and (c) coupling medium 3 are used.

measured dielectric properties of water to curves obtained from the model.

When the coupling media are used, received signals are weaker compared to the air case due to the propagation loss. Therefore, two LNAs would be employed in order to improve the signal's strength. Under this situation, the noise power was calculated and presented in Table 2 . We can see that in comparison with the air case, the noise contributed by the antenna and the LNA network have higher values. This is due to the use of one more LNA and the change of the antenna performance.

Figure 8 (b) shows the strongest measured signal when coupling medium 1 is used and the waveform looks very different from that in Figure 8(a). The SNRs of the strongest and the weakest received signals are shown in Figure 11 when the three different coupling media are used. The results suggest that the use of the coupling media shifts the optimal working band to the low frequency end. As the conductivity of the coupling media increases, the SNR difference between the strongest and the weakest signals becomes larger due to a higher loss.

A simple experiment was carried out in order to verify such an effect. We used ethanol as the coupling medium because its dielectric properties are close to those of the coupling media we considered in the simulations. Figure 12(a) plots the measured dielectric properties of the ethanol. In 


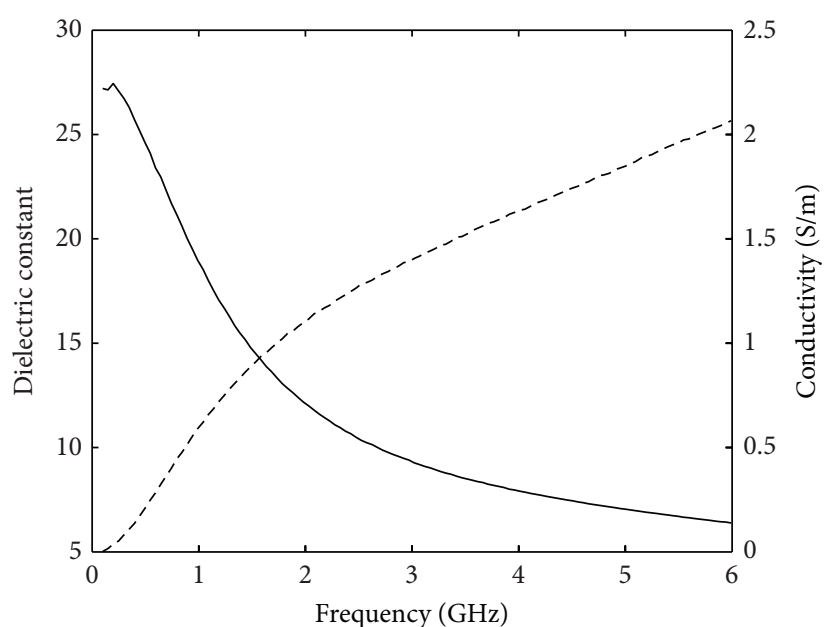

(a)

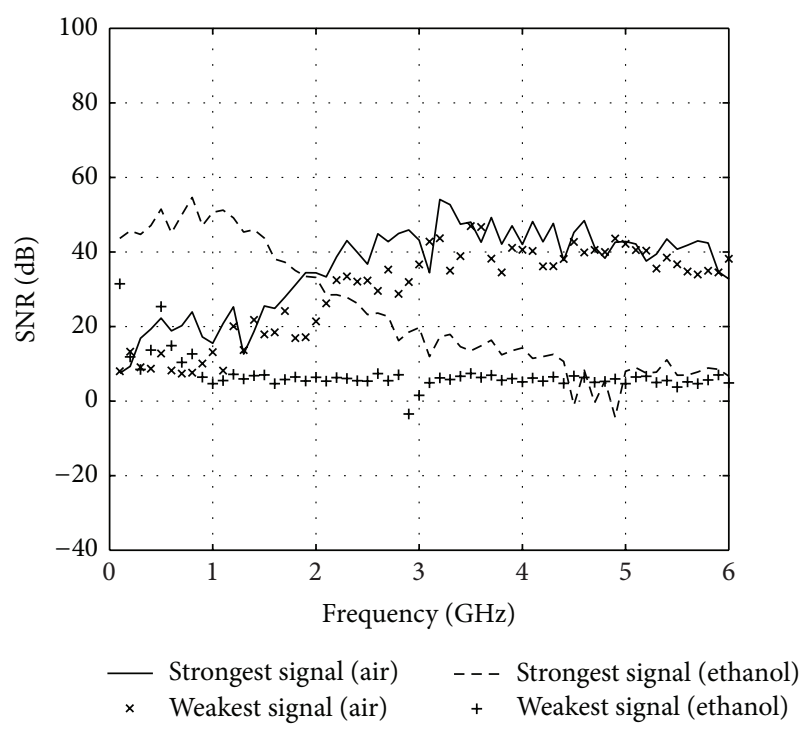

(b)

FIGURE 12: (a) The dielectric properties of the ethanol measured with a dielectric probe and (b) the comparison of the measurement SNRs when the antenna array is surrounded with air and ethanol, respectively.

order to reduce the amount of ethanol to be used, a twodimensional circular antenna array composed of twenty monopoles was used in the experiment. With the pulse generator connected to one of the antennas, we recorded the signals received by the other antennas by using a sampling oscilloscope when the antenna array was surrounded with air and ethanol, respectively. The SNRs of the strongest and weakest signals were calculated from several repetitive measurements according to [6] and shown in Figure 12(b) for both cases. It can be seen that the results demonstrate the same effect as that based on simulations.

Therefore, the use of a coupling medium could better the measurement accuracy in the frequency range of interest but puts higher requirements on the dynamic range of the system. A variable gain amplifier (VGA) may be used to improve the SNR of weak signals and the gain of the SNR is dependent on the noise performance of the VGA. For too weak a signal, the gain is very limited because the front-end noise would dominate the system noise.

4.3. SNR due to Timing Jitter. Aside from the effect of the voltage noise, timing jitter also plays a very important role in determining the SNR when it comes to the measurement of a UWB time domain signal. Although the jitter error can be compensated [19], it is interesting to investigate its effect in comparison with the voltage noise.

Simulations are performed to estimate SNRs due to different levels of timing jitter and the jitter is modeled as a normally distributed random process. The two signals presented in Figure 8 are sampled with a jitter clock according to (9) and the sampling rate is $50 \mathrm{GSa} / \mathrm{s}$. The standard deviation as defined in (11) is calculated from $N=100$ sampled signals, and the SNRs obtained from (12) are shown in Figure 13 for two different jitter levels.
The results indicate that when the jitter increases, the degradation of the SNR is about the same for all the frequency components except for the low frequency region in Figure 13(a) and the high frequency region in Figure 13(b), where smaller changes are observed. This exception is because of the poorer agreement between the averaged amplitude spectrum $\overline{Y(f)}$ and the amplitude spectrum of the actual jitter-free sampled signal, which is ultimately attributed to the lower signal level in these regions.

As mentioned in Section 3.2, the total timing jitter of the system is around 2 ps and by comparing Figure 13 with Figures 9 and 11, we can conclude that the jitter-resulted SNR is comparable to that due to the voltage noise for the strongest signal. Jitter plays a less important role in determining the SNR of a weaker signal in comparison with the voltage noise since jitter-induced noise is dependent on the signal level.

\section{Conclusion}

We have presented a time domain microwave imaging system and evaluated its noise performance under different conditions. The results suggest that the antenna array and the coupling media play important roles in determining the systems performance. Therefore, in order to obtain the optimal performance, these two factors need to be considered in the hardware design of a time domain system for medical imaging.

For an effective sampling rate of $50 \mathrm{Gsa} / \mathrm{s}$, a SNR higher than $25 \mathrm{~dB}$ can be obtained in the frequency range of interest when the most lossy coupling medium is used. The obtained data is based on the assumption that the T/H and the ADC are perfectly matched. Although this is not true in practice, the mismatch loss can be made small.

While the noise level of the presented system is comparable to that of a commercial sampling oscilloscope [6], its 


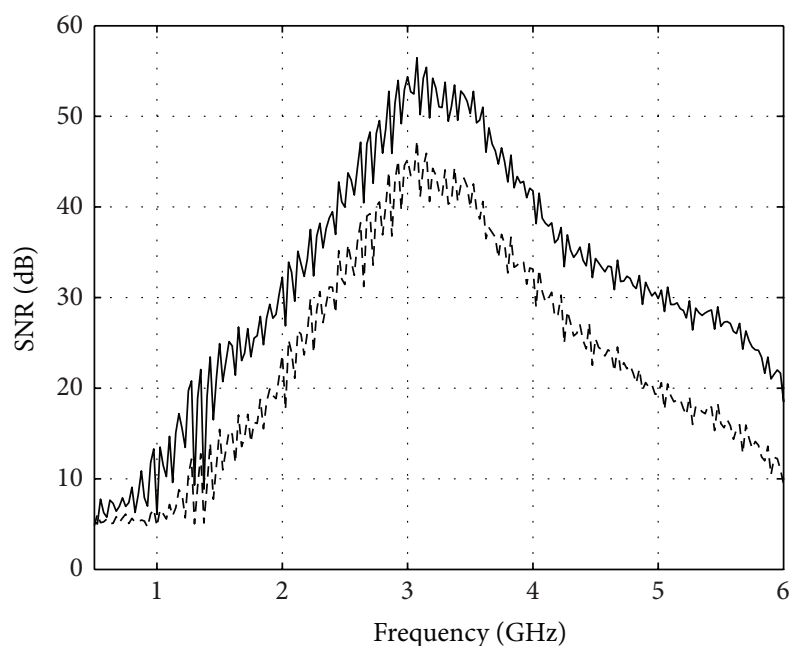

1 ps rms jitter - - 3 ps rms jitter

(a)

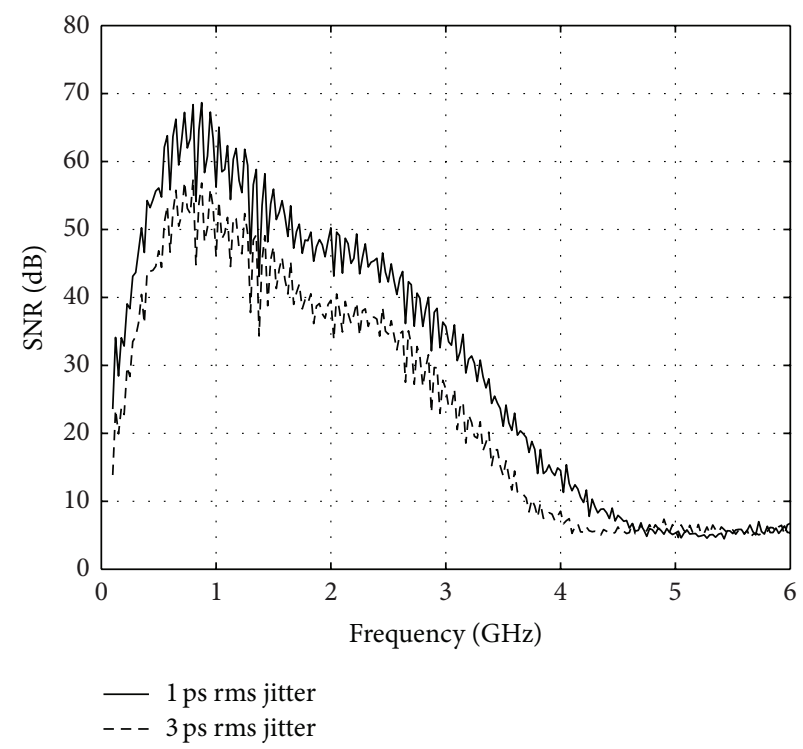

(b)

FIGURE 13: The SNR of (a) the signal presented in Figures 8(a) and 8(b) the signal presented in Figure 8(b) due to timing jitter.

measurement speed is much higher. For example, with the same effective sampling rate, it takes $50 \mathrm{~ms}$ to record a time domain signal with the length of $40 \mathrm{~ns}$ by using the sampling oscilloscope and with the presented system, the measurement time is less than $0.5 \mathrm{~ms}$.

The system is now under implementation and its performance will be tested and reported in the future.

\section{Acknowledgments}

This work was supported in part by VINNOVA within the VINN Excellence Center Chase and in part by SSF within the Strategic Research Center Charmant. The computations were performed on C3SE computing resources.

\section{References}

[1] A. Fhager, P. Hashemzadeh, and M. Persson, "Reconstruction quality and spectral content of an electromagnetic time-domain inversion algorithm," IEEE Transactions on Biomedical Engineering, vol. 53, no. 8, pp. 1594-1604, 2006.

[2] M. Klemm, I. J. Craddock, J. A. Leendertz, A. Preece, and R. Benjamin, "Radar-based breast cancer detection using a hemispherical antenna array: experimental results," IEEE Transactions on Antennas and Propagation, vol. 57, no. 6, pp. 1692-1704, 2009.

[3] S. M. Salvador and G. Vecchi, "Experimental tests of microwave breast cancer detection on phantoms," IEEE Transactions on Antennas and Propagation, vol. 57, no. 6, pp. 1705-1712, 2009.

[4] E. C. Fear, J. Bourqui, C. Curtis, D. Mew, B. Docktor, and C. Romano, "Microwave breast imaging with a monostatic radarbased system: a study of application to patients," IEEE Transactions on Microwave Theory and Techniques, vol. 61, no. 5, pp. 2119-2128, 2013.

[5] J. C. Y. Lai, C. B. Soh, E. Gunawan, and K. S. Low, "UWB microwave imaging for breast cancer detection: experiments with heterogeneous breast phantoms," Progress in Electromagnetics Research M, vol. 16, pp. 19-29, 2011.

[6] X. Zeng, A. Fhager, P. Linner, M. Persson, and H. Zirath, "Experimental investigation of the accuracy of an ultrawideband timedomain microwave-tomographic system," IEEE Transactions on Instrumentation and Measurement, vol. 60, no. 12, pp. 39393949, 2011.

[7] J. Schoukens, R. M. Pintelon, and Y. J. Rolain, "Broadband versus stepped sine FRF measurements," IEEE Transactions on Instrumentation and Measurement, vol. 49, no. 2, pp. 275-278, 2000.

[8] M. Kahrs, "50 years of RF and microwave sampling," IEEE Transactions on Microwave Theory and Techniques, vol. 51, no. 6, pp. 1787-1805, 2003.

[9] Y. Yang and A. E. Fathy, "Development and implementation of a real-time see-through-wall radar system based on FPGA," IEEE Transactions on Geoscience and Remote Sensing, vol. 47, no. 5, pp. 1270-1280, 2009.

[10] Programmable delay chip MC100EP196. On semiconductor.

[11] D. M. Pozar, Microwave Enginnering, John Wiley Sons, 3rd edition, 2005.

[12] C. K. S. Miller, W. C. Daywitt, and M. G. Arthur, "Noise standards, measurements, and receiver noise definitions," Proceddings of the IEEE, vol. 55, no. 6, pp. 865-877, 1967.

[13] T. Y. Otoshi, "The effect mismatched components on microwave noisetemperature calibrations," IEEE Transactions on Microwave Theory and Techniques, vol. 16, no. 9, pp. 675-686, 1968.

[14] G. Gonzalez, Microwave Transistor Amplifiers: Analysis and Design, Prentice Hall, 2nd edition, 1997.

[15] X. Zeng, A. Fhager, M. Persson, P. Linner, and H. Zirath, "Accuracy evaluation of ultrawideband time domain systems for microwave imaging," IEEE Transactions on Antennas and Propagation, vol. 59, no. 11, pp. 4279-4285, 2011.

[16] B. N. Taylor and C. E. Kuyatt, "Guidelines for evaluating and expressing the uncertainty of NIST measurement results," NIST Technical Note 1297, NIST, 1994. 
[17] M. Lazebnik, M. Okoniewski, J. H. Booske, and S. C. Hagness, "Highly accurate debye models for normal and malignant breast tissue dielectric properties at microwave frequencies," IEEE Microwave and Wireless Components Letters, vol. 17, no. 12, pp. 822-824, 2007.

[18] M. Lazebnik, D. Popovic, L. McCartney et al., "A large-scale study of the ultrawideband microwave dielectric properties of normal, benign and malignant breast tissues obtained from cancer surgeries," Physics in Medicine and Biology, vol. 52, no. 20, pp. 6093-6115, 2007.

[19] P. D. Hale, C. M. Wang, D. F. Williams, K. A. Remley, and J. D. Wepman, "Compensation of random and systematic timing errors in sampling oscilloscopes," IEEE Transactions on Instrumentation and Measurement, vol. 55, no. 6, pp. 2146-2154, 2006. 

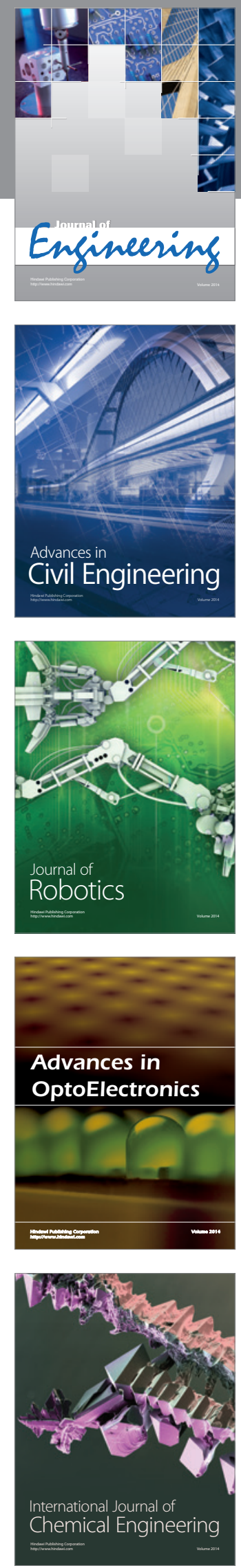

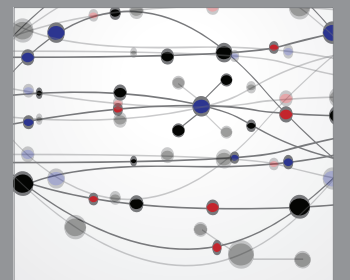

The Scientific World Journal
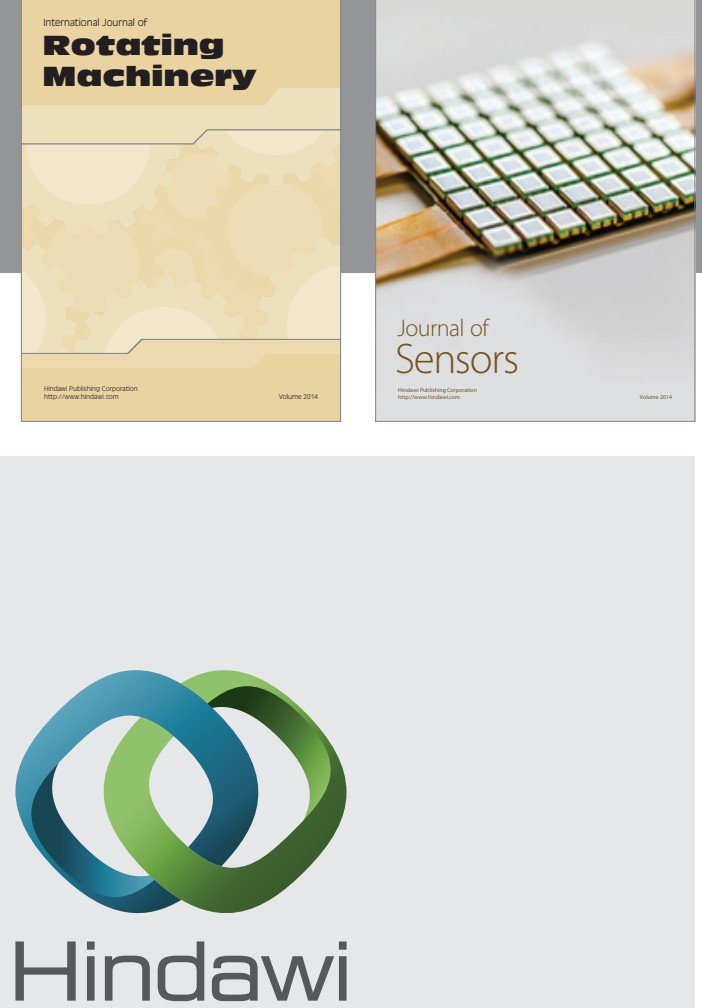

Submit your manuscripts at http://www.hindawi.com
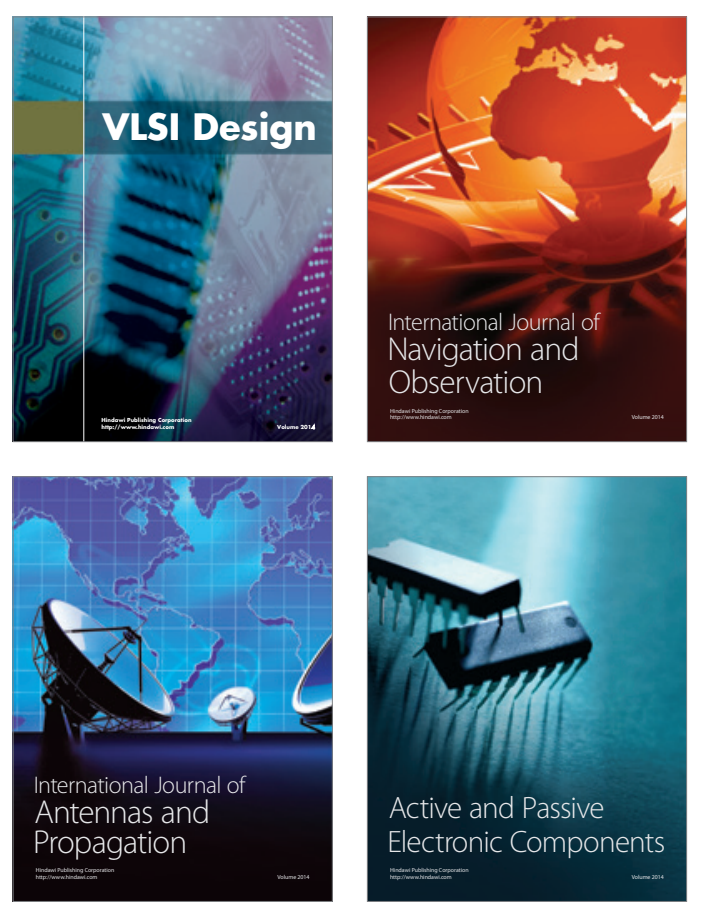
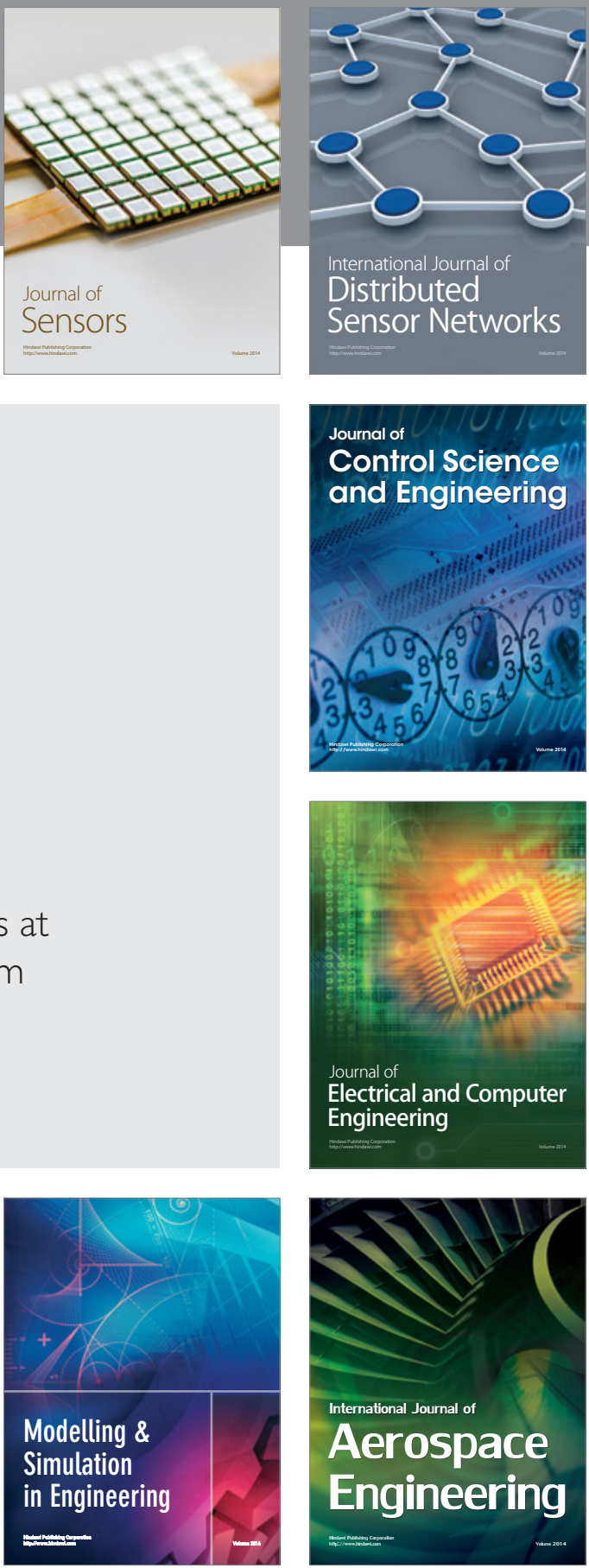

Journal of

Control Science

and Engineering
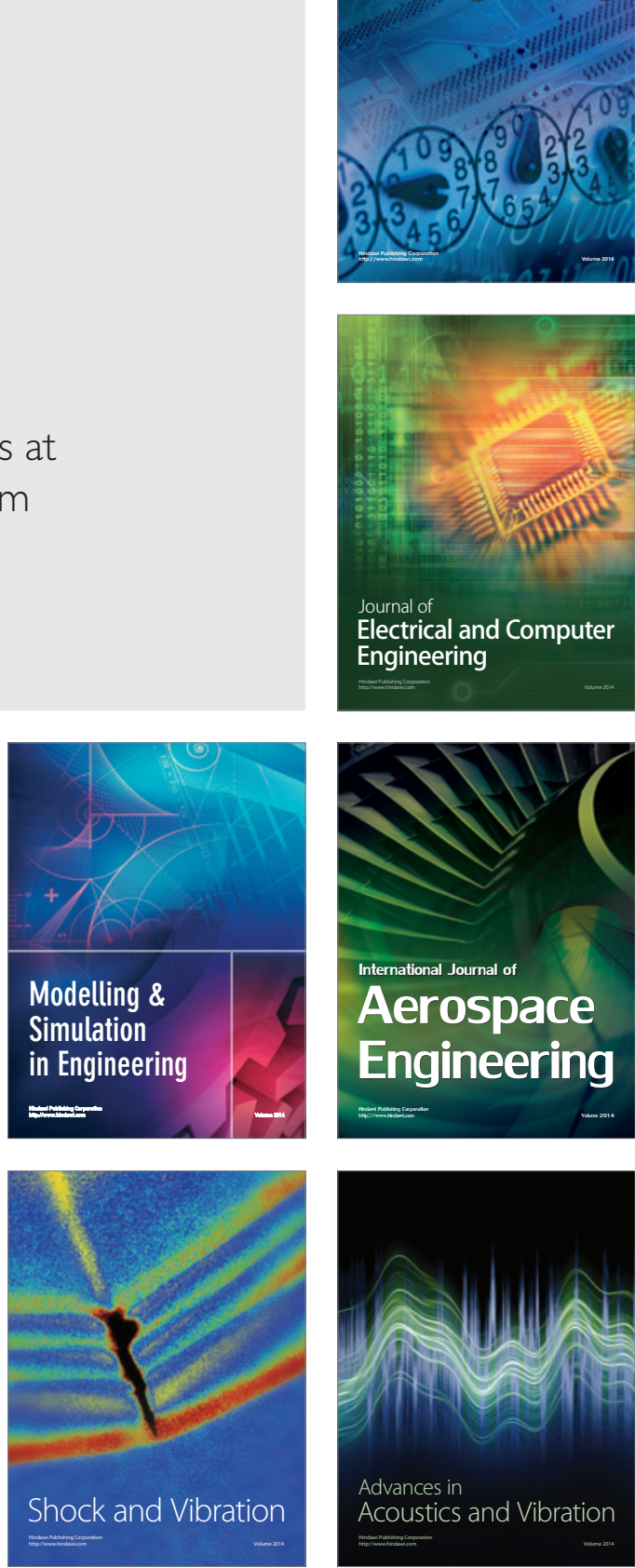